Proceedings of the 1996 IEEE

International Conference on Robotics and Automation

Minneapolis, Minnesota - April 1996

\title{
Fault Tolerance for Kinematically Redundant Manipulators: Anticipating Free-Swinging Joint Failures
}

\author{
James D. English and Anthony A. Maciejewski \\ Purdue University \\ 1285 Electrical Engineering Building \\ West Lafayette, Indiana 47907-1285
}

\begin{abstract}
Fault tolerance is an important design criterion for robotic systems operating in hazardous or remote environments. This article addresses the issue of tolerating a free-swinging joint failure by focusing on how to best configure a slow-moving manipulator before a failure. Three scalar measures of fault susceptibility are defined using joint torques/forces, acceleration, and swing angles. Minimizing these measures is an approach to achieving fault tolerance, and for this, algorithms to calculate their gradients are given. The formulas are valid for general $n$-link manipulators.
\end{abstract}

\section{INTRODUCTION}

Robots that operate in remote or hazardous environments must be used in a manner that reflects the implications of failure scenarios on system performance $[1,2,3]$. Kinematically redundant robots have been proposed for use in such environments due to their dexterity before a failure and ability to continue operation after a failure $[4,5,6]$. A crucial component of any system designed to tolerate failures is the ability to detect and address different failure modes [7]. Much of this previous work has focused on failures that are modeled as locked joints, either because the failure directly results in an inability to move or because brakes are applied to prevent unpredictable behavior.

In contrast, the study of free-swinging failures is still in its infancy and presents fresh problems and additional possibilities for usefulness after a failure [8]. The term free-swinging failure refers to a hardware or software fault in a robotic manipulator that causes the loss of torque (or force) on a joint. Examples include a ruptured seal on a hydraulic actuator, the loss of electric power and brakes on an electric actuator, and a mechanical failure in a drive system. After a free-swinging failure, the failed joint moves freely under the influence of external forces and gravity, hence the descriptive label.

The work described here addresses the issue of how

This work was supported by Sandia National Laboratories under contract number AL-3011 and by a NASA graduate student research fellowship (grant number NGT9-2). to best configure a slow-moving kinematically redundant manipulator in anticipation of a free-swinging failure. Manipulators used in hazardous or remote environments are typically slow moving. Kinematic redundancy allows the best configuration to be found by establishing fault tolerance as a secondary criterion to be met without affecting the end-effector task. Three secondary criteria will be developed for this purpose, each addressing a different aspect of a failure: torque/force, acceleration, or swing angle.

\section{A Mathematical Framework for Establish- ing Failure-Susceptibility Measures}

The method for reducing the likelihood or negative consequences of a failure is this: A scalar measure of failure susceptibility is defined as a function of the joint variables, then it is minimized using the manipulator's kinematic redundancy. The approach to defining an overall measure will be to first establish for each joint a measure of susceptibility to a failure of that joint alone, then combine these in a meaningful way to form the comprehensive scalar measure.

Let $k_{i}(\mathbf{q})$ be the failure-susceptibility measure of joint $i$ alone. Then, for an $n$-degree-of-freedom manipulator, a column of joint measures, $\mathbf{k}(\mathbf{q})$, is formed as follows:

$$
\mathbf{k}(\mathbf{q})=\left[\begin{array}{llll}
k_{1} & k_{2} & \cdots & k_{n}
\end{array}\right]^{T} .
$$

Let $f_{k}$ represent the comprehensive failuresusceptibility measure. Then for positive semidefinite weighting matrix $\mathbf{W}_{k}$, the form of $f_{k}$ to be used in this work is

$$
f_{k}(\mathbf{q})=\mathbf{k}(\mathbf{q})^{T} \mathbf{W}_{k} \mathbf{k}(\mathbf{q}) .
$$

To reduce the effects of an impending free-swinging joint failure, $f_{k}$ is minimized. Several widely known methods of optimizing secondary cost functions under the constraint of completing a primary task have been presented. The augmented-Jacobian technique 
$[9,10]$ can be used to track a desired value of the secondary criterion function. For precise tracking of critical points, the extended-Jacobian technique is appropriate [11]. And to track a local minimum or maximum, the gradient-projection method can be used $[12,13]$. These techniques require knowledge of the gradient of the function.

Application of the chain rule to (2) gives the gradient of $f_{k}$ as

$$
\nabla f_{k}=2[D \mathbf{k}]^{T} \mathbf{W}_{k} \mathbf{k}
$$

where

$$
[D \mathbf{k}]=\left[\begin{array}{llll}
\nabla k_{1} & \nabla k_{2} & \cdots & \nabla k_{n}
\end{array}\right]^{T} .
$$

With this, $\nabla f_{k}$ is established as a function of the jointwise measures ( $k_{i}$ 's) and their gradients ( $\nabla k_{i}$ 's). Sections III, IV, and $V$ to follow will develop values for the $k_{i}$ 's (each addressing a different failure aspect) and methods to calculate their gradients.

\section{A Torque-Based Measure}

In this section, a fault-susceptibility measure will be given that is based on the joint torques/forces (hereafter "joint torque" will be used to denote either torque or force for rotational or prismatic joints, respectively). A joint-torque-based measure is appropriate in that the torques at a manipulator's joints are related to both the effect and likelihood of a failure. Should joint $i$ undergo a free-swinging failure during operation, the torque on joint $i$ is precisely the instantaneous forcedomain change induced by the failure. It is related to the joint acceleration after a failure (this relationship will be detailed in Section IV), and if it is zero the failure will have no immediate effect. Moreover, a joint under less torque is less likely to fail. (The load to induce a failure in a mechanical part is reasonably assumed to have a normal distribution [14].) Thus, minimizing the joint-torque-based failure-susceptibility measure will both limit the effect of a failure and help prevent the onset of a failure.

A shortcoming of the torque-based measure is that a low-torque solution may result in a high swing angle for a rotational joint after a failure. This issue will be addressed in Section V.

\section{A. Definition of the Measure, $f_{g}(\mathbf{q})$}

Joint torques are functions of the manipulator's motion and configuration. For a viscous friction model, this relationship is expressed mathematically as follows:

$$
\tau=\mathbf{M}(\mathbf{q}) \ddot{\mathbf{q}}+\mathbf{C}(\mathbf{q}, \dot{\mathbf{q}}) \dot{\mathbf{q}}+\mathbf{V}(\mathbf{q}) \dot{\mathbf{q}}+\mathbf{g}(\mathbf{q}) .
$$

Here, $\tau$ is the vector of joint torques; $q$ is the vector of joint positions; $\mathbf{M}(\mathbf{q})$ is the manipulator inertia matrix;
$\mathbf{C}(\dot{\mathbf{q}}, \mathbf{q})$ is the matrix specifying centrifugal and Coriolis effects; $\mathbf{V}(\mathbf{q})$ is the viscous-friction matrix; and $\mathbf{g}(\mathbf{q})$ is the vector of joint torques due to gravity.

Local optimization of the total torque given by (5) has been addressed [15], but it was found that this approach has regions of inherent instability [16]. The task of globally optimizing a function of the torques from (5) over a path has also been addressed $[17,18]$, but such global optimizations are computationally complex and not appropriate for on-line control. For a slow-moving manipulator, however, an approximation can be made that allows stable on-line optimization. The torques can be reduced to those of the static case, where $\dot{\mathbf{q}}$ and $\ddot{\mathbf{q}}$ vanish, and (5) becomes

$$
\tau=\mathbf{g}(\mathbf{q}) \text {. }
$$

This gives the vector of joint torques needed to counter gravity in a stationary manipulator, and each individual torque forms a measure through its magnitude of the susceptibility to a free-swinging failure of its joint.

Using $\mathbf{k}=\mathbf{g}$ in (2) gives $f_{g}$, the scalar failuresusceptibility measure for the torque-based approach. Here $\mathbf{W}_{k}=\mathbf{W}_{g}$ is a positive semidefinite matrix that weights the relative importance of the joint torques (see Section VI for an example).

\section{B. Calculating the Gradient, $\nabla f_{g}$}

For $\mathbf{k}=\mathbf{g}$, (3) establishes the gradient of the overall cost function as a function of the joint torques (g) and the torques' gradients $(D \mathrm{~g})$. The remainder of this section describes a method for finding these quantities.

\section{B.1 Joint $i$ Rotational}

When joint $i$ is rotational, the torque $g_{i}$ can be calculated as follows:

$$
g_{i}=\left(\hat{z}_{i-1} \times \vec{s}_{i-1}^{*}\right) \cdot \vec{g},
$$

where $\hat{z}_{\ell}$ is the unit vector along joint $\ell+1$, the $\mathrm{z}$-axis of the $\ell^{\text {th }}$ Denavit-Hartenberg (D-H) coordinate frame; $\vec{s}_{\ell}^{*}$ is the first moment of inertia of the composite rigid body formed by links $\ell+1$ through $n$ referred to the origin of $\mathrm{D}-\mathrm{H}$ frame $\ell$; and $\vec{g}$ is the gravity vector in the upward direction.

Let $M_{i}$ be the composite mass of links $i$ through $n$, calculated for all joint types as follows:

$$
M_{i}=m_{i}+M_{i+1} ; M_{n}=m_{n} .
$$

Here $m_{i}$ is the mass of link $i$. Using these values, the composite first-moment-of-inertia vector $\vec{s}_{i}^{*}$ as expressed in the $i^{\text {th }} \mathrm{D}-\mathrm{H}$ frame can be calculated for all joint types as follows:

$$
\vec{s}_{i}^{*}={ }^{i} \mathbf{R}_{i+1}\left(\vec{s}_{i+1}^{*}+\vec{s}_{i+1}+M_{i+1} \vec{p}_{i \rightarrow i+1}\right) ; \vec{s}_{n}^{*}=\overrightarrow{0}
$$


Here, ${ }^{i} \mathbf{R}_{i+1}$ is the $3 \times 3$ rotation matrix representing D-H frame $i+1$ in frame $i$; $\vec{s}_{\ell}$ is the first-moment-ofinertia vector for link $\ell$ referred to and expressed in its own D-H frame; and $\vec{p}_{i \rightarrow \ell}$ is the vector from the origin of $\mathrm{D}-\mathrm{H}$ frame $i$ to the origin of frame $\ell$, expressed in frame $\ell$.

The gradient of $g_{i}$ has entries $\frac{\partial g_{i}}{\partial q_{j}}$. For joint $i$ rotational, using (7), the values of these entries are given by

$$
\frac{\partial g_{i}}{\partial q_{j}}=\left(\left[\frac{\partial}{\partial q_{j}} \hat{z}_{i-1}\right] \times \vec{s}_{i-1}^{*}+\hat{z}_{i-1} \times\left[\frac{\partial}{\partial q_{j}} \vec{s}_{i-1}^{*}\right]\right) \cdot \vec{g}
$$

provided $\frac{\partial}{\partial q_{j}} \hat{z}_{i-1}$ and $\frac{\partial}{\partial q_{j}} \vec{s}_{i-1}^{*}$ are found with respect to the base frame and $\vec{g}$ is constant in the base frame. This allows calculation of the gradient once the vector partial derivatives are found. Equation (10) is given in coordinate-free form, and after calculation of $\frac{\partial}{\partial q_{j}} \hat{z}_{i-1}$ and $\frac{\partial}{\partial q_{j}} \vec{s}_{i-1}^{*}$ it can be evaluated in any frame.

\section{B.2 Joint $i$ Prismatic}

When joint $i$ is prismatic, the force $g_{i}$ is a function only of the orientation of the joint (as compared to the dependence on the first moment of inertia when joint $i$ is rotational). The value $g_{i}$ is now given by

$$
g_{i}=M_{i} \hat{z}_{i-1} \cdot \vec{g}
$$

and the elements of the gradient are

$$
\frac{\partial g_{i}}{\partial q_{j}}=M_{i}\left(\frac{\partial}{\partial q_{j}} \hat{z}_{i-1} \cdot \vec{g}\right)
$$

provided $\frac{\partial}{\partial q_{j}} \hat{z}_{i-1}$ is found with respect to the base frame and $\vec{g}$ is constant in the base frame. Like (10), this can be evaluated in any frame once the vector partial derivatives are found.

\section{Calculation of $\frac{\partial}{\partial q_{j}} \hat{z}_{i}$ and $\frac{\partial}{\partial q_{j}} \vec{s}_{i}^{*}$}

The elements of $\nabla g_{i}$ can be calculated directly from (10) or (12) only after $\frac{\partial}{\partial q_{j}} \hat{z}_{i}$ and $\frac{\partial}{\partial q_{j}} \vec{s}_{i}^{*}$ have been evaluated. When joint $j$ is rotational, $\frac{\partial}{\partial q_{j}} \hat{z}_{i}$ and $\frac{\partial}{\partial q_{j}} \vec{s}_{i}^{*}$ can be found as follows:

$$
\begin{array}{rlrl}
\frac{\partial}{\partial q_{j}} \hat{z}_{i} & =\hat{z}_{j-1} \times \hat{z}_{i} & j \leq i \\
& =\hat{0} & j>i \\
\frac{\partial}{\partial q_{j}} \vec{s}_{i}^{*} & =\hat{z}_{j-1} \times \vec{s}_{j-1}^{*} & j>i \\
& =\hat{z}_{j-1} \times \vec{s}_{i}^{*} & j \leq i,
\end{array}
$$

and when joint $j$ is prismatic,

$$
\begin{array}{rlrl}
\frac{\partial}{\partial q_{j}} \hat{z}_{i} & =\overrightarrow{0} & \\
\frac{\partial}{\partial q_{j}} \vec{s}_{i}^{*} & =\overrightarrow{0} & & j \leq i \\
& =M_{j} \hat{z}_{j-1} & j>i .
\end{array}
$$

\section{An AcCeleration-Based Measure}

The last section presented a fault-susceptibility measure based on the stationary joint torques. Among the justifications for this was joint torque's relationship to joint acceleration after a failure. In this section, the jointwise function will be precisely the acceleration after a failure. Though this new measure does not have the failure-prevention properties of the torque-based approach, it relates more accurately to the immediate failure dynamics. If failed joint acceleration is low, more time is available to compensate for the failure before significant arm motion. This principle, and the method, applies to both rotational and prismatic joints. As in the torque-based case, a stationary manipulator is assumed as an approximation to a slow-moving manipulator.

\section{A. Definition of the Measure, $f_{a}(\mathbf{q})$}

The velocity of an inertial body cannot change instantaneously. So, at the moment of failure for a stationary manipulator, the joint rates do not change, $\dot{q}$ remains 0 , and equation (5) becomes

$$
\tau=\mathbf{M}(\mathbf{q}) \ddot{\mathbf{q}}+\mathbf{g}(\mathbf{q}),
$$

where, for failed joint $i$,

$$
\tau_{i}=0,
$$

and since a stationary prefailure manipulator is assumed,

$$
\ddot{q}_{j}=0, \quad j \neq i \text {. }
$$

With (18) and (19), (17) gives the following scalar equation for $a_{i}=\ddot{q}_{i}$, the acceleration of the failed joint:

$$
0=M_{i i} a_{i}+g_{i},
$$

where $M_{i i}$ is diagonal entry $i$ of $\mathbf{M}$. Since the manipulator inertia matrix is positive definite [19], $M_{i i}$ is strictly positive, and the following is always valid:

$$
a_{i}=-\frac{g_{i}}{M_{i i}} \text {. }
$$

With this, the column vector of joint measures is

$$
\mathbf{a}=\left[\begin{array}{llll}
a_{1} & a_{2} & \cdots & a_{n}
\end{array}\right]^{T} .
$$

Using $\mathbf{k}=\mathbf{a}$ and $\mathbf{W}_{k}=\mathbf{W}_{a}$, a positive semidefinite weighting matrix, the framework of Section II establishes $f_{a}$, the scalar failure-susceptibility measure for the acceleration-based approach. Note this is an anticipatory measure (the acceleration is manifest only if a failure occurs) and not a measure of a current physical phenomenon as was the torque-based measure. 


\section{B. Calculating the Gradient, $\nabla f_{a}$}

With $\mathbf{k}=\mathbf{a},(3)$ allows calculation of the gradient of $f_{a}$ as a function of the postfailure accelerations (a) and their gradients $(D \mathbf{a})$.

The entries of each $\nabla a_{i}$ (which forms row $i$ of $D \mathbf{a}$ ) can be found by applying the quotient rule to (21):

$$
\frac{\partial a_{i}}{\partial q_{j}}=\frac{-\frac{\partial g_{i}}{\partial q_{j}} M_{i i}+\frac{\partial M_{i i}}{\partial q_{j}} g_{i}}{M_{i i}^{2}}
$$

Equations (21) and (23) allow calculation of $\mathbf{a}$ and $D \mathbf{a}$ once $M_{i i}, \frac{\partial M_{i i}}{\partial q_{j}}, g_{i}$, and $\frac{\partial g_{i}}{\partial q_{j}}$ are known. The joint torque $\tau_{i}=g_{i}$ can be calculated using (7) for joint $i$ rotational or (11) for joint $i$ prismatic. The partial derivatives of $g_{i}$ can be calculated using (10) for joint $i$ rotational or (12) for joint $i$ prismatic. This leaves $M_{i i}$ and $\frac{\partial M_{i i}}{\partial q_{j}}$.

\section{Calculation of the Diagonal Entries of the Mass Ma- trix and Their Gradients}

The methods for calculating $M_{i i}$ and $\frac{\partial M_{i i}}{\partial q_{j}}$ will be broken down into cases for prismatic versus rotational joints and inboard versus outboard variables for the gradients.

\section{C.1 The Diagonal Entries}

When joint $i$ is rotational, $M_{i i}$ is given by

$$
M_{i i}=\hat{z}_{i-1} \cdot \mathbf{J}_{i-1}^{*} \hat{z}_{i-1},
$$

where $\mathbf{J}_{\ell}^{*}$ is the composite rigid-body inertia of links $\ell+1$ through $n$ referred to $\mathrm{D}-\mathrm{H}$ frame $\ell$. It can be calculated recursively as follows (adapted from [20]):

$$
\begin{gathered}
\mathbf{J}_{i}^{*}=\stackrel{{ }^{i} \mathbf{R}_{i+1}\left(\mathbf{J}_{i+1}^{*}+\mathbf{J}_{i+1}-\mathbf{P}_{i \rightarrow i+1}\left(\mathbf{S}_{i+1}^{*}+\mathbf{S}_{i+1}\right)-\right.}{\left.\left(\mathbf{S}_{i+1}^{*}+\mathbf{S}_{i+1}+M_{i+1} \mathbf{P}_{i \rightarrow i+1}\right) \mathbf{P}_{i \rightarrow i+1}^{i}\right)^{i} \mathbf{R}_{i+1}^{T}} ; \\
\mathbf{J}_{n}^{*}=\mathbf{0} .
\end{gathered}
$$

The matrix $\mathbf{S}_{\ell}$ is the cross-product matrix for $\vec{s}_{\ell}, \mathbf{S}_{\ell}^{*}$ is the cross-product matrix for $\vec{s}_{\ell}^{*}$, and $\mathbf{P}_{i \rightarrow \ell}$ is the crossproduct matrix for $\vec{p}_{i \rightarrow \ell}$. Matrices without a preceding superscript are expressed in their frame of definition $(\ell$ for $\mathbf{J}_{\ell}, \mathbf{J}_{\ell}^{*}, \mathbf{S}_{\ell}, \mathbf{S}_{\ell}^{*}$, and $\mathbf{P}_{\boldsymbol{i} \rightarrow \ell}$ ).

When joint $i$ is prismatic, $M_{i i}$ is simply the composite mass of links $i$ through $n$; i.e.,

$$
M_{i i}=M_{i},
$$

with the composite mass $M_{i}$ calculated using (8).

\section{C.2 The Gradients}

For joints $i$ and $j$ rotational, $i<j$, from (24),

$$
\frac{\partial M_{i i}}{\partial q_{j}}=\hat{z}_{i-1} \cdot \frac{\partial}{\partial q_{j}} \mathbf{J}_{i-1}^{*} \hat{z}_{i-1},
$$

where the fact that $\hat{z}_{i-1}$ is inboard from $q_{j}$ and therefore does not change with $q_{j}$ was used. Taking the partial derivative of $\mathbf{J}_{i-1}^{*}$ with respect to $q_{j}$ gives, in coordinate-free form,

$$
\begin{aligned}
\frac{\partial}{\partial q_{j}} \mathbf{J}_{i-1}^{*}= & \mathbf{Z}_{j-1} \mathbf{J}_{j-1}^{*}+\mathbf{P}_{i-1 \rightarrow j-1}\left(\mathbf{S}_{j-1}^{*} \mathbf{Z}_{j-1}-\mathbf{Z}_{j-1} \mathbf{S}_{j-1}^{*}\right) \\
& -\mathbf{J}_{j-1}^{*} \mathbf{Z}_{j-1}+\left(\mathbf{S}_{j-1}^{*} \mathbf{Z}_{j-1}-\mathbf{Z}_{j-1} \mathbf{S}_{j-1}^{*}\right) \mathbf{P}_{i-1 \rightarrow j-1}
\end{aligned}
$$

where $\mathbf{Z}_{\ell}$ is the cross-product matrix for $\hat{z}_{\ell}$. Substituting (29) into (28), exploiting the symmetry of $J_{j-1}^{*}$, and simplifying gives the following:

$$
\begin{aligned}
\frac{\partial M_{i i}}{\partial q_{j}}=2\left(\hat{z}_{i-1} \times \vec{p}_{(i-1)-(j-1)} \cdot\left(\vec{s}_{j-1}^{*} \times\left(\hat{z}_{j-1} \times \hat{z}_{i-1}\right)-\right.\right. \\
\left.\left.\hat{z}_{j-1} \times\left(\vec{s}_{j-1}^{*} \times \hat{z}_{i-1}\right)\right)+\left(\hat{z}_{i-1} \times \hat{z}_{j-1}\right) \cdot \mathbf{J}_{j-1}^{*} \hat{z}_{i-1}\right) .
\end{aligned}
$$

This can be efficiently calculated in frame $j-1$.

For joints $i$ and $j$ rotational, $i \geq j, M_{i i}$ is constant for changing $q_{j}$, and thus

$$
\frac{\partial M_{i i}}{\partial q_{j}}=0
$$

For joint $i$ rotational and joint $j$ prismatic, $i<j$, taking the partial derivative of $\mathbf{J}_{i-1}^{*}$ with respect to $q_{j}$ (now a sliding variable) gives, in coordinate-free form,

$$
\frac{\partial}{\partial q_{j}} \mathbf{J}_{i-1}^{*}=-\mathbf{P}_{i-1 \rightarrow j-1}\left[\frac{\partial}{\partial q_{j}} \mathbf{S}_{j-1}^{*}\right]-\left[\frac{\partial}{\partial q_{j}} \mathbf{S}_{j-1}^{*}\right] \mathbf{P}_{i-1 \rightarrow j-1} .
$$

Using $\frac{\partial}{\partial q_{j}} \mathbf{S}_{j-1}^{*}=M_{j} \mathbf{Z}_{j-1}$ with (32) in (28), which is valid for all types of joint $j$, gives

$$
\frac{\partial M_{i i}}{\partial q_{j}}=2 M_{j}\left(\hat{z}_{i-1} \times \hat{z}_{j-1}\right) \cdot\left(\hat{z}_{i-1} \times \vec{p}_{(i-1) \rightarrow(j-1)}\right) ;
$$

this can be efficiently calculated in frame $j-1$.

For joint $i$ rotational and joint $j$ prismatic, $i>j$, the composite rigid-body inertia of links $i$ through $n$ is not changed by the value of joint variable $j$, and thus

$$
\frac{\partial M_{i i}}{\partial q_{j}}=0 \text {. }
$$

For prismatic joint $i$, all $j$, the composite mass of links $i$ through $n$ is not changed by the value of joint variable $j$, and thus, from (27),

$$
\frac{\partial M_{i i}}{\partial q_{j}}=0 \text {. }
$$

\section{A Swing-Angle-Based Measure}

In this section, the failure-susceptibility measure will be based on the angle through which a failed rotational joint moves after a failure, that is, the angle between the prefailure configuration and the settled, postfailure configuration. This is defined as the swing angle. 


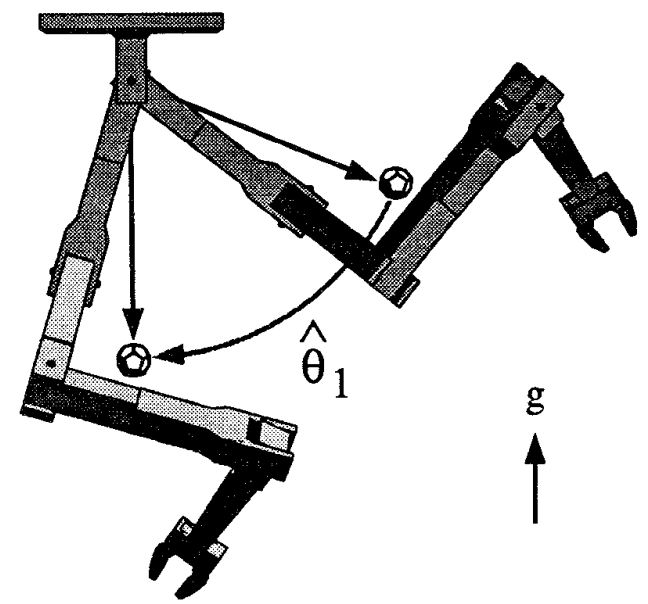

Fig. 1. The swing angle for joint one, $\hat{\theta}_{1}$. Shown here is a manipulator before (upper right) and after (lower) a failure of the first joint. The center of mass of the manipulator is represented by the small polyhedron.

When it is small, a failure will produce a displacement that will, in a relative sense, be small, and when it is zero, a failure will have no effect (for the given assumptions). The expectation is that with a small swing angle, the manipulator is less likely to cause secondary damage to itself or its environment. This measure is for rotational joints only (prismatic joints do not settle through friction, but hit stops), and, again, a stationary manipulator is assumed as an approximation to slow motion.

If the environment is well known, configurationspace analysis of the workspace could be used to specify a range of swing angles that would not induce collision. No assumptions about the environment, however, are made for for this work, and the approach will be to reduce the magnitude of the swing angles. A shortcoming of a swing-angle based measure is that it provides a limited amount of information on the Cartesian motion of the manipulator-this is the subject of current research.

\section{A. Definition of the Measure, $f_{\hat{\theta}}(\mathbf{q})$}

The swing angle $\hat{\theta}_{i}$ is the angle through which failed joint $i$ moves to find its resting position after a failure. The resting position is that for which the center of mass of the portion of the manipulator outboard from the failed joint is at its lowest position relative to the gravitational field. This is illustrated for a failure of the base joint in Fig. 1.

With the definitions of $\hat{z}_{\ell}, \vec{s}_{\ell}^{*}$, and $\vec{g}$ as given in Section III, the angle through which joint $i$ would swing were it to fail is given by the angle between the projections of $\vec{s}_{i-1}^{*}$ and $-\vec{g}$ onto the plane perpendicular to $\hat{z}_{i-1}$ (the axis of rotation). This can be calculated as the angle between $\left(\vec{s}_{i-1}^{*} \times \hat{z}_{i-1}\right)$ and $\left(\hat{z}_{i-1} \times \vec{g}\right)$ (see Fig. 2); i.e.,

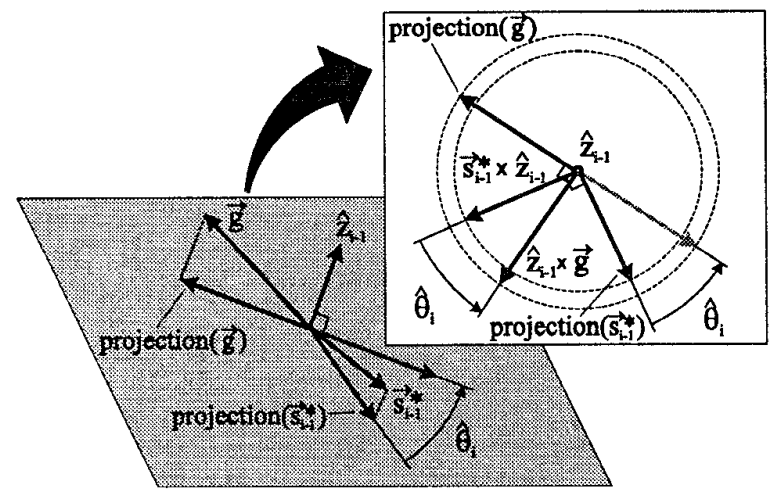

Fig. 2. The swing angle is the angle between the projection of $\vec{s}_{i-1}^{*}$ and the negative of the projection of $\vec{g}$. It is equal to the angle between $\vec{s}_{i-1}^{*} \times \hat{z}_{i-1}$ and $\hat{z}_{i-1} \times \vec{g}$.

$$
\begin{aligned}
& \cos \left(\hat{\theta}_{i}\right)=\frac{\left(\vec{s}_{i-1}^{*} \times \hat{z}_{i-1}\right) \cdot\left(\hat{z}_{i-1} \times \vec{g}\right)}{\left\|\vec{s}_{i-1}^{*} \times \hat{z}_{i-1}\right\|\left\|\hat{z}_{i-1} \times \vec{g}\right\|} \\
& \sin \left(\hat{\theta}_{i}\right)=\frac{\hat{z}_{i-1} \cdot\left(\vec{g} \times \vec{s}_{i-1}^{*}\right)}{\left\|\vec{s}_{i-1}^{*} \times \hat{z}_{i-1}\right\|\left\|\hat{z}_{i-1} \times \vec{g}\right\|} .
\end{aligned}
$$

Provided neither $\vec{s}_{i-1}^{*}$ nor $\vec{g}$ is parallel to $\hat{z}_{i-1}$, equations (36) and (37) give

$$
\hat{\theta}_{i}=\operatorname{Atan} 2\left[\hat{z}_{i-1} \cdot\left(\vec{g} \times \vec{s}_{i-1}^{*}\right),\left(\vec{s}_{i-1}^{*} \times \hat{z}_{i-1}\right) \cdot\left(\hat{z}_{i-1} \times \vec{g}\right)\right],
$$

where the range of Atan 2 is $-\pi$ to $\pi$; otherwise, from $(7)$, the torque on joint $i$ is zero, and since a stationary manipulator is assumed,

$$
\hat{\theta}_{i}=0 \text {. }
$$

The jointwise measure for use within the framework established in Section II will be the swing angle. The column vector of joint measures will be given by

$$
\hat{\theta}=\left[\begin{array}{llll}
\hat{\theta}_{1} & \hat{\theta}_{2} & \cdots & \hat{\theta}_{n}
\end{array}\right]^{T} .
$$

Using $\mathbf{k}=\hat{\theta}$ and $\mathbf{W}_{k}=\mathbf{W}_{\hat{\theta}}$, a positive semidefinite weighting matrix, the framework of Section II establishes $f_{\hat{\theta}}$, the scalar failure-susceptibility measure for the swing-angle-based approach.

\section{B. Calculating the Gradient, $\nabla f_{\hat{\theta}}$}

The gradient of $f_{\hat{\theta}}$ can be calculated using ( 3 ), which, for $\mathbf{k}=\hat{\theta}$, establishes the gradient of the measure as a function of the swing angles $(\hat{\theta})$ and their gradients $(D \hat{\theta})$.

If $\vec{s}_{i-1}^{*}$ or $\vec{g}$ is parallel to $\hat{z}_{i-1}$, the gradient of $\hat{\theta}_{i}$ is either 0 or undefined and should be set to 0 . Otherwise, from (38), the entries of $\nabla \hat{\theta}_{i}$ are calculated as follows:

$$
\frac{\partial \hat{\theta}_{i}}{\partial q_{j}}=\frac{1}{u_{i, 1}^{2}+u_{i, 2}^{2}}\left(\frac{\partial u_{i, 1}}{\partial q_{j}} u_{i, 2}-\frac{\partial u_{i, 2}}{\partial q_{j}} u_{i, 1}\right),
$$


where

$$
u_{i, 1}=\hat{z}_{i-1} \cdot\left(\vec{g} \times \vec{s}_{i-1}^{*}\right)
$$

and

$$
u_{i, 2}=\left(\vec{s}_{i-1}^{*} \times \hat{z}_{i-1}\right) \cdot\left(\hat{z}_{i-1} \times \ddot{g}\right) .
$$

Equation (41) is valid for all values of $\hat{\theta}_{i}$ over $-\pi$ to $\pi$.

\section{Calculation of $\frac{\partial}{\partial q_{j}} u_{i, 1}$ and $\frac{\partial}{\partial q_{j}} u_{i, 2}$}

When all vector quantities are expressed in the base frame and the gravity vector there is constant, (42) and (43) give the following:

$$
\frac{\partial u_{i, 1}}{\partial q_{j}}=\frac{\partial}{\partial q_{j}} \hat{z}_{i-1} \cdot\left(\vec{g} \times \vec{s}_{i-1}^{*}\right)+\hat{z}_{i-1} \cdot\left(\vec{g} \times \frac{\partial}{\partial q_{j}} \vec{s}_{i-1}^{*}\right)
$$

and

$$
\begin{gathered}
\frac{\partial u_{i, 2}}{\partial q_{j}}=\left(\frac{\partial}{\partial q_{j}} \vec{s}_{i-1}^{*} \times \hat{z}_{i-1}+\vec{s}_{i-1}^{*} \times \frac{\partial}{\partial q_{j}} \hat{z}_{i-1}\right) \cdot\left(\hat{z}_{i-1} \times \vec{g}\right) \\
+\left(\vec{s}_{i-1}^{*} \times \hat{z}_{i-1}\right) \cdot\left(\frac{\partial}{\partial q_{j}} \hat{z}_{i-1} \times \vec{g}\right),
\end{gathered}
$$

where $\frac{\partial}{\partial q_{j}} \hat{z}_{i-1}$ and $\frac{\partial}{\partial q_{j}} \vec{s}_{i-1}^{*}$ can be calculated using (13), (14), (15), and (16).

\section{EXAMPles}

To illustrate the concepts in this article, a threelink, vertically planar manipulator will be used because it is easy to visualize. The techniques and all equations are valid for general manipulators. The example manipulator's link lengths are unity; the link masses are unity; and the center of mass of each link is at the link center. The link inertias are modeled as thin rods. The task will be end-effector positioning only (i.e., orientation is not considered), and with this perspective, the manipulator has one degree of kinematic redundancy.

For the examples, the focus will be on a single joint failure. To achieve this, the choice of $\mathbf{W}_{k}$ to be used in (2) is a $3 \times 3$ matrix with a one in the appropriate diagonal position and all other entries zero. For example, to focus on joint two, the following $\mathbf{W}_{k}$ is used:

$$
\mathbf{W}_{k}=\left[\begin{array}{lll}
0 & 0 & 0 \\
0 & 1 & 0 \\
0 & 0 & 0
\end{array}\right]
$$

\section{A. Torque- and Acceleration-Based Examples}

Using the type of weighting matrix exemplified by (46) with the gradient projection method gave the results shown in Fig. 3 (with no failure occurring) for the torque-based and acceleration-based approaches with a rectangular end-effector trajectory.

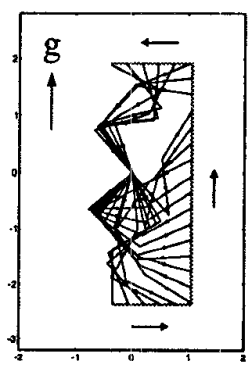

Torque Based Joint 1

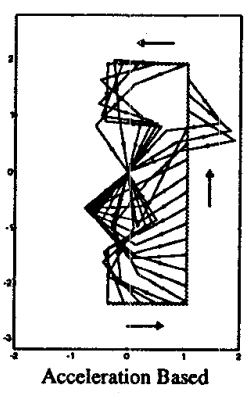

Joint 1

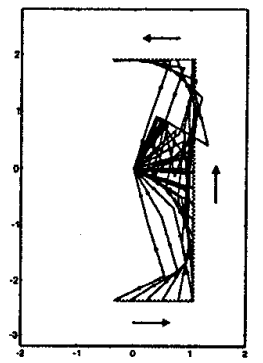

Torque Based Joint 2

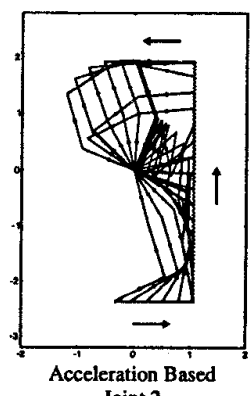

Joint 2

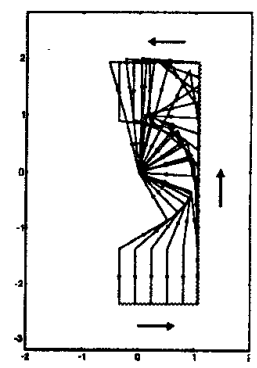

Torque Based Joint 3

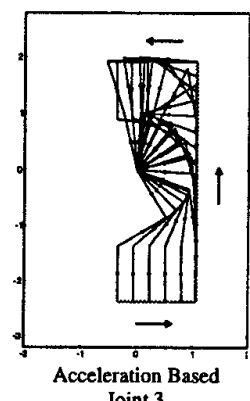

Joint 3
Fig. 3. Following a rectangular trajectory beginning in the lower left-hand corner and ending in the upper left, for the torque- and acceleration-based methods with focus on each of the joints. Link centers of nass are shown as points.

For the torque-based approach, the method tends to maintain the composite center of mass of the links outboard from the focus joint either above or below the joint. This is partially true for the acceleration-based approach, with a shared tendency to increase the moment of inertia about the focus joint. The difference can be seen by comparing the joint-one-based cases in Fig. 3. Near the top of the long edge of the trajectory rectangle, joint three is withdrawn for the torquebased case (reducing the torque) but extended for the acceleration-based case (increasing the moment of inertia at the expense of increased torque).

Where zero torque is achievable, the two methods may give the same configurations. This is the case for the portion of the trajectory along the bottom segment of the rectangle for all cases in Fig. 3. However, the two methods may not give the same solution even when both have a criterion-function value of zero, as is the case along the top segment of the rectangle for the jointone and joint-two cases. This shows the existence of multiple zero torque/acceleration solution sets.

For the joint-three cases, the two methods give the same solution for this example (see Fig. 3). This is true in general for focus on the last joint, and follows from the fact that the moment of inertia of the last link about the last joint is static and therefore minimizing the acceleration squared is equivalent to minimizing the torque squared as a direct consequence of (21). 


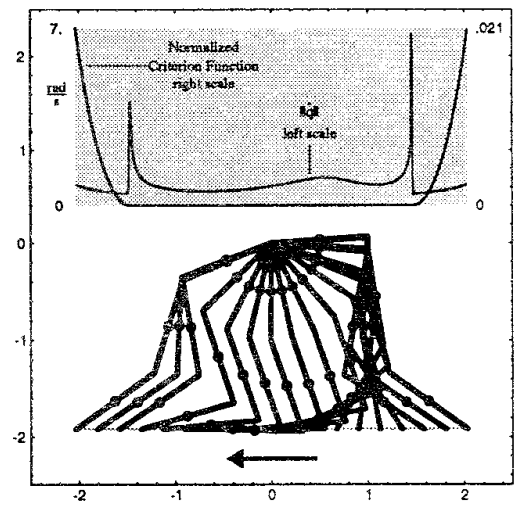

Fig. 4. Following a horizontal trajectory from right to left while minimizing the joint-two swing-angle-based measure. The normalized (globally largest value $=1$ ) value of the criterion function (the joint-two swing angle squared) and the joint-rate norm (for a $10 \mathrm{~s}$ constant-velocity trajectory) are plotted in the gray region versus the $\mathrm{x}$-coordinate of the end effector.

\section{B. Swing-Angle-Based Examples}

Focusing on the joint-two swing angle over a linear trajectory gave the results of Fig. 4 (with no failure occurring). The cost-function was zero over most of the the trajectory, and where zero was not achievable, the swing-angle magnitude was minimized, as is evidenced by the tendency of the last two links to stay under the second joint. Had joint two failed while the arm slowly traversed the trajectory, the deviation of the arm from the desired path would have been small-the worst case criterion function value is $2.1 \%$ of its global maximum.

Also in Fig. 4, the joint-rate norm is plotted versus the end-effector $\mathrm{x}$-coordinate for a $10 \mathrm{~s}$ constantvelocity trajectory, and the spikes show that the manipulator experiences rapid motion at two points along the path. These points correspond to occurrences of algorithmic singularities-the manipulator's configuration at these points is not a differentiable function of end-effector position under minimizing control. The nature of the failure-susceptibility measure at and near the right-hand point is detailed in Fig. 5.

Rapid manipulator motion is undesirable and voids the slow-moving assumption. Reducing the end-effector trajectory speed could prevent this [21], but if constant velocity is desired, a solution is to restrict null-space motion to reduce the joint rates without sacrificing endeffector velocity tracking. For example, in simulations, capping the joint-rate norm at $2.0 \mathrm{rad} / \mathrm{s}$ for the trajectory of Fig. 4 caused only a minor change in the criterion function with no error in the task. If error in the task were permissible, a damped-least-squares type of solution could be used to limit the joint velocity.

\section{Postfailure Workspaces}

For the example manipulator, regions exist in the workspace where, for the case of focus on a single
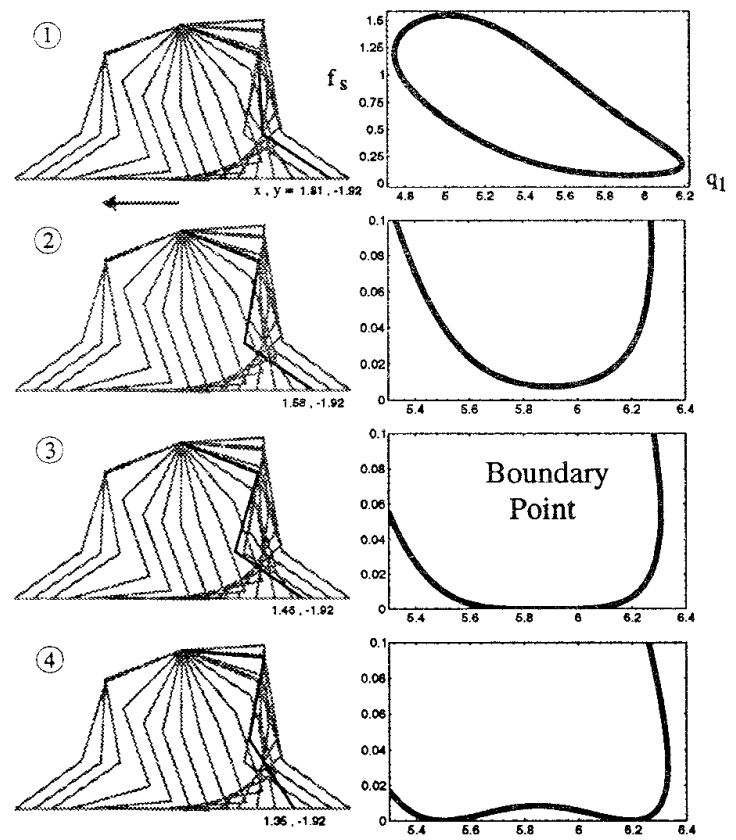

Fig. 5. An illustration of the splitting-local-minimum phenomenon based on the joint-two swing-angle-based measure. The manipulator is following a horizontal trajectory while locally minimizing the swing-angle-based measure (the same path as in Fig. 4). Four plots are shown of the swing-angle-based criterion function versus the first joint angle in radians (measured from horizontal) for configurations giving a desired end point. Each plot corresponds to the end point of the black arm to its left. The first plot shows the range of all possible values, while the second through fourth plots show a subset. The local minimum is well defined in plots one and two, but becomes poorly defined in plot three, with the end effector at the boundary of the zerocriterion-function region-the system experiences an algorithmic singularity at this point. In the fourth plot, the minimum has split (the black arm corresponds to the right-hand minimum), and the end-effector is now well within the zero-criterion-function region of the workspace. (See Fig. 6.)

joint, all three fault-susceptibility measures can be zero. These regions correspond to the stable ${ }^{1}$ postfailure workspaces, i.e., the sets of poses that can be reached after a failure. The stable postfailure workspaces for the example manipulator are shown in Fig. 6. Any point reachable after a failure of joint $i$ is also reachable by a healthy manipulator with all three joint- $i$-based criterion functions zero $\left(\hat{\theta}_{i}=0\right.$ implies no motion after a failure implies $a_{i}=0$ implies $g_{i}=0$ ). For example, it was upon the arm's passing into the medium-gray region of Fig. 6 that the criterion function first became zero in Fig. 4 corresponding to the boundary point of Fig. 5. The arm in this configuration is shown against the regions in Fig. 6.

\footnotetext{
${ }^{1} \mathrm{~A}$ zero-torque configuration is stable if the composite center of mass of the links outboard from the failed joint lie below (with respect to the gravitational field) the line coinciding with the axis of the failed joint.
} 


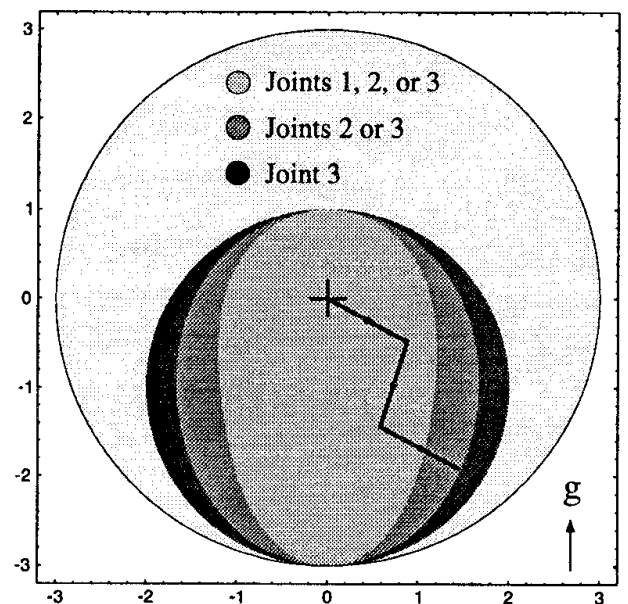

Fig. 6. Regions of the workspace where all three single-jointbased measures can be zero. These correspond to the stable workspaces after a failure. The central light-gray region is reachable after any joint failure. The medium-gray region is reachable after a failure of the second or third joint. And the outer dark region is reachable after a failure of the third joint. The large circular light-gray region is the healthy workspace. The arm shown here corresponds to the third black arm of Fig. 5 and has zero torque/postfailure acceleration/swing angle for the second joint.

\section{SUMmary}

This article defined three cost functions which quantitatively reflected the susceptibility of a manipulator to a free-swinging joint failure. The torque-based function measured failure likelihood and force-domain effects; the acceleration-based function measured immediate failure dynamics; and the swing-angle-based function measured susceptibility to secondary damage after a failure. For use in minimization methods, algorithms were given for calculating the gradient of each cost function for a general spatial manipulator. These were used with the gradient-projection technique to show the methods' usefulness for a three-link vertically planar example manipulator.

These three measures address diverse aspects of a robotic system's susceptibility to a failure. They can be used independently or together. When employed to control the motion of manipulators in remote or hazardous environments, they have the potential to reduce the likelihood and negative consequences of a failure and thereby expand the general usefulness of robotic manipulators.

\section{ReferenCes}

[1] B. Christensen, W. Drotning, and S. Thunborg, "Modelbased, sensor-directed remediation of underground storage tanks," J. Robotic Sys., vol. 9, no. 2, pp. 145-159, 1992.

[2] R. Colbaugh and M. Jamshidi, "Robot manipulator control for hazardous waste-handling applications," J. Robotic Sys., vol. 9, no. 2, pp. 215-250, 1992.
[3] E. Wu, J. Hwang, and J. Chladek, "Fault tolerant joint development for the space shuttle remote manipulator system:Analysis and experiment," in Proc. Fourth Int. Symp. Robot. Manufacturing (ISRAM '92), pp. 505-510, (Sante Fe, New Mexico), Nov. 11-13 1992.

[4] A. A. Maciejewski, "Fault tolerant properties of kinematically redundant manipulators," in Proc. 1990 IEEE Int. Conf. Robot. Auto., pp. 638-642, (Cincinnati, OH), May 13181990.

[5] C. J. J. Paredis, W. K. F. Au, and P. K. Khosla, "Kinematic design of fault tolerant manipulators," Computers Elec. Eng.: An Int. J., vol. 20, no. 3, pp. 211-220, May 1994.

[6] C. L. Lewis and A. A. Maciejewski, "Dexterity optimization of kinematically redundant manipulators in the presence of failures," Camputers Elec. Eng.: An Int. J., vol. 20, no. 3, pp. 273-288, May 1994.

[7] M. L. Visinsky, J. R. Cavallaro, and I. D. Walker, "A dy. namic fault tolerance framework for remote robots," IEEE Trans. Robot. Auto., vol. 11, no. 4, pp. 477-490, Aug. 1995.

[8] H. Arai and S. Tachi, "Position control of a manipulator with passive joints using dynamic coupling," IEEE Trans. Robot. Auto., vol. 7, no. 4, pp. 528-534, 1991.

[9] O. Egeland, "Task-space tracking with redundant manipula. tors," IEEE J. Robot. Auto., vol. RA-3, no. 5, pp. 471-475, Oct. 1987.

[10] H. Seraji, "Configuration control of redundant manipulators: Theory and implementation," IEEE Trans. Robot. Auto., vol. 5, no. 4, pp. 472-490, Aug. 1989.

[11] J. Baillieul, "Kinematic programming alternatives for redundant manipulators," in Proc. 1985 IEEE Int. Conf. Robot. Auto., pp. 722-728, (St. Louis, MO), Mar. 25-28 1985.

[12] A. Liégeois, "Automatic supervisory control of the configuration and behavior of multibody mechanisms," IEEE Trans. Sys. Man Cybernet., vol. SMC-7, no. 12, pp. 868-871, Dec. 1977.

[13] C. A. Klein and C. H. Huang, "Review of pseudoinverse control for use with kinematically redundant manipulators," IEEE Trans. Sys. Man Cybernet., vol. SMC-13, no. 2, pp. 245-250, Mar./Apr. 1983.

[14] R. C. Johnson, Optimum Design of Mechanical Elements, John Wiley and Sons, New York, 1980.

[15] J. M. Hollerbach and K. C. Suh, "Redundancy resolution of manipulators through torque optimization," IEEE J. Robot. Auto., vol. RA-3, no. 4, pp. 308-316, Aug. 1987.

[16] A. A. Maciejewski, "Kinetic limitations on the use of redundancy in robotic manipulators," IEEE Trans. Robot. Auto., vol. 7, no. 2, pp. 205-210, Apr. 1991.

[17] K. C. Suh and J. M. Hollerbach, "Local versus global torque optimization of redundant manipulators," in Proc. 1987 IEEE Int. Conf. Robot. Auto., pp. 619-624, (Raleigh, NC), Mar. 31-April 31987.

[18] Y. Nakamura and H. Hanafusa, "Optimal redundancy control of robot manipulators," Int. J. Robot. Res., vol. 6, no. 1, pp. 32-42, Spring 1987.

[19] L. T. Wang and B. Ravani, "Recursive computations of kinematic and dynamic equations for mechanical manipulators," IEEE J. Robot. Auto., vol. RA-2, no. 3, pp. 124-131, Sep. 1985.

[20] A. Fijany and A. K. Bejczy, "An efficient algorithm for computation of manipulator inertia matrix," J. Robotic Sys., vol. 7, no. 1, pp. 57-80, 1990.

[21] J. M. Hollerbach, "Dynamic scaling of manipulator trajectories," ASME J. Dyn. Sys. Meas. Contr., vol. 106, pp. 102106, March 1984. 